\title{
Fuzzy Fault Tree Analysis based on Interpretive Structure Model and Binary Connection Numbers
}

\author{
Honghua Sun, Hongxia Chen", Qingyang Li, and Xudong Chen \\ School of Mechanical Engineering, Inner Mongolia University of Technology, Hohhot, 010051, China
}

\begin{abstract}
Building a fault tree and calculating the sequence of bottom events importance degree are key steps in fault diagnosis. Two improvements are made to the fuzzy fault tree in this study. The first is building the fault tree using Interpretive Structure Modeling (ISM) technology. The second is transforming triangular fuzzy numbers into binary connection numbers (BCN) through the uncertainty theory of set pair analysis, where the certainty coefficient is determined by the median of the triangular fuzzy number and the uncertainty coefficient is determined by the interval value described by the upper and lower limitations. The formula of failure probability of the top event and the formula of probability importance of the bottom event are deduced with the binary connection number. This method reduces the calculation amount. A case study is carried out to verify the feasibility and effectiveness of the method.
\end{abstract}

Keywords: fault tree; triangular fuzzy number; interpretive structure modeling (ISM); binary connection number (BCN)

(Submitted on October 8, 2018; Revised on November 17, 2018; Accepted on December 15, 2018)

(c) 2019 Totem Publisher, Inc. All rights reserved.

\section{Introduction}

A fault tree is an analysis method to improve system reliability. The logic relation diagram is drawn by analyzing the factors that affect a system's failures (including hardware, software, environment, human factors, etc.). The fault tree analysis method can determine the possible combinations cause and failure probability of the system failure, and then measures can be taken to prevent system failures. Fault trees describe the logical relationship between the basic events that lead to the failure of the system by using prescribed events, logic gates, and other symbols.

Failure Mode Effect and Criticality Analysis (FMECA) and Fault Tree Analysis (FTA) are both ways to express fault causality and retrieve fault information. Hu et al. explored the correlation of information between FMECA and FTA and combined the two methods. C \# language and SQL database were utilized to develop computer-aided software [1]. Du et al. integrated FTA and FMECA methods, and a corresponding FTAMCA system was built up based on VB and ACCESS [2]. Tao et al. combined the disadvantages of FMECA and FTA, presented the automatic transform algebra between the FMECA and FTA of sub fault tree, and then established the computer aided dynamic fault tree method [3]. Xu et al. proposed the fault tree automatic modeling method based on the avionics system architecture model. The safety analysis model was constructed by adding safety properties to the system design model and embedding an assertion mechanism of the advanced formal language Al-taRica to describe the fault transition process [4]. Yang systematically studied the fault diagnosis and analysis model based on the FMECA generated in the design phase of product, and a new method of automatic generation of fault tree analysis system based on FMECA was proposed. Compared with manual construction, this method can obtain the fault tree with complete diagnosis knowledge [5]. In order to systematically support system faults modeling and formal analysis, Huang et al. proposed a fault tree generation method based on fault configuration. [6]. Li et al. established the fault tree by analyzing the fault cause of the clutch and brake through fishbone diagrams. The fault tree was analyzed qualitatively by binary decision diagrams to calculate the importance of the minimum cut set and determine the rule priority, and the problem of rule conflict was solved [7]. $\mathrm{Gu}$ et al. used the interpretive structure model (ISM) to

* Corresponding author.

E-mail address: chenhx@imut.edu.cn 
analyze the interrelation between the risk factors of the project, and the direct causes affecting the collapse of the tunnel project were obtained, and then the fault tree model was established [8]. In order to study the influence factors of controlled flight on terrain of general aviation, Sun et al. proposed the targeted prevention measure, and 30 controlled flight into terrain events were analyzed by using the element event analysis method. The paired comparison analysis of the influence factors was carried out using the interpretation structure (ISM) [9]. To scientifically reveal the inherent law of Controlled Flight into Terrain (CFIT) inducement for preventing CFIT accidents, Mei et al. used the Interpretative Structural Model to analyze the relationship of CFIT inducement [10]. In view of the complex fault mechanism of complex manufacturing systems, which is hierarchical, latent, causal, and fragile, Xia et al. put forward the DEMATEL-ISM, a quantitative analysis method for critical fault factors of the processing workshop [11].

Huang et al. put forward a method of bottom event sorting based on BDD. In addition to considering the influence of the number of layers of events in the fault tree on sorting, the method also takes into account the factors such as repeated events, adjacent events, and the number of events contained in logic gates. The above four indicators jointly determine the priority of bottom event sequencing [12]. Xu et al. and Li et al. thought that triangular fuzzy numbers are also commonly used mathematical tools in solving decision problems. Based on the possible degree formulas of the comparison of the triangular fuzzy numbers and the ranking formulas of the complementary judgment matrix, the decision plans are ranked and selected [13-14]. Zhao et al. and Li et al. used the fuzzy number median method to obtain the fuzzy importance degree and order of each basic event [15-16]. By utilizing the theories of set pair analysis and statistical, the interval fuzzy number is transformed into connection number in the form of "the mean and variance", which retains the certainty and uncertainty in interval triangular fuzzy decision-making information [17]. By using the uncertainty systems theory in the set pair analysis, the median, and the interval of values prescribed by the upper and lower bounds of triangular fuzzy numbers, the triangular fuzzy numbers are transformed into connection numbers, and then the decision model is given. The attribute values with the triangular fuzzy number are converted into connection numbers with the form of "average + deviation", and the sort of uncertainty is analyzed by using the " $i$ " different values of connection numbers [18-19]. Lu et al. used the connection number in multi-attribute decision-making and proposed a new method of the " $i$ " value. [20]. Formation and diagnosis of turbine vibration usually is affected by many uncertain factors. Based on the uncertainty of power equipment fault diagnosis process, the uncertainty analysis connection mathematical theory was used for fault diagnosis of turbines by Zhao [21].

Fault trees are used to analyze the causality between faults, and the ISM is also used to establish the hierarchy between causality, so this paper applies the method of ISM to the establishment of fault trees. There are many methods for ordering the probability importance of bottom events. BCN is applied to conduct evaluation problems, but no one has applied BCN to the solution of probability importance of fuzzy fault trees previously. Hence, this paper studies the following two aspects: using the ISM to build fault trees and applying the BCN to triangular fuzzy numbers to calculate the probability importance of the bottom event.

This paper is organized as follows. Section 2 introduces the basic principle and steps of the interpretive structure modeling. In Section 3, the binary connection number is defined, the procedure of how to transform triangle fuzzy numbers into binary connection numbers is described, and the reliability formula by binary connection number is deduced. In Section 4 , a case study is given to verify the effectiveness of using ISM and BCN. Section 5 provides the conclusions and future works.

\section{The Steps to Build a Model by ISM}

The ISM is a determining technology of the whole structure of the system based on various creative techniques. By exploring the elements of the system and defining the significance of the correlation between the elements, the concrete relationship between the elements based on the binary relation is given. Moreover, the structure model of a complex system is established by digraph and matrix, which are easy to understand and easy to deal with. The technology of interpretive structure modeling was developed by Warfield in 1973 to analyze complex socio-economic problems [22]. The flow chart of the fault tree is set up by using ISM, as shown in Figure 1. The modeling steps are as follows.

Step 1 Establish the adjacency matrix A of direct binary relations between elements. "1" in a matrix denotes the binary relationship between row elements and column elements. The binary relationship includes influence, causality, inclusion, membership, or comparison. The binary relationship is defined as causality when applied to a fault tree. The adjacency matrix $A$ and the unit matrix $I$ are combined to form the $(A+I)$ matrix:

$$
A+I=\left[a_{i j}\right]_{n \times n}
$$




$$
a_{i j}=\left\{\begin{array}{l}
1, \text { i element has binary relations to } j \text { element } \\
0, i \text { element has no binary relations to } j \text { element }
\end{array}\right.
$$

$a_{i j}=1$ means $i$ element has binary relation to $j$ element, and $a_{i j}=0$ means $i$ element has no binary relation to $j$ element.

Step 2 The reachable matrix $M$ is calculated according to the $(A+I)$ matrix. The $M$ matrix contains direct, indirect, and self to self-binary relations.

$$
(A+I)^{2} \neq(A+I)^{3} \neq \cdots \neq(A+I)^{r-1} \neq(A+I)^{r}=M
$$

Step 3 According to the reachable matrix $M$, the number of " 1 " in each row is calculated. The position of the elements is rearranged according to the number of row " 1 " from less to more, and the unit matrix of the largest order is drawn.

Step 4 Remove the binary relation of the leapfrogging, and transform the multi-hierarchical digraph into interpretive structure modeling on the basis of understanding the practical meaning of the elements.

Step 5 Transform the interpretive structure modeling into a fault tree.

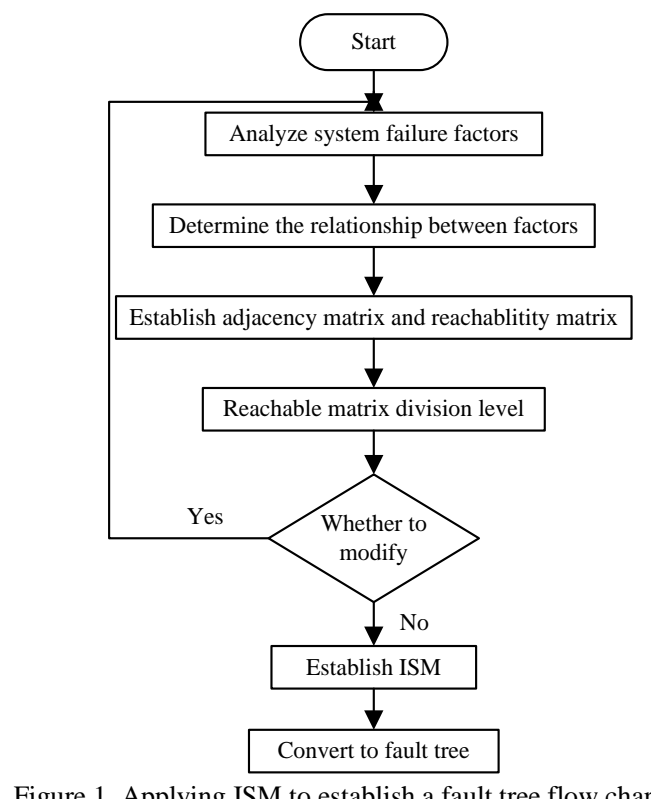

\section{Transform Triangular Fuzzy Number into Connection Numbers}

\subsection{Triangular Fuzzy Number}

In general, the probability of a bottom event is obtained from a reliability manual or an empirical manual, but in some practical systems there are a large number of uncertainties, such as natural climate and human operations, which have an impact on the probability of the bottom event. Therefore, the probability of these bottom events does not have accurate values that can be counted. Even if the probability of the bottom event is obtained, these values are not certain because of the incidental complexity of the fault. If there is no statistical data, the determination of the probability of bottom events is generally based on the method of expert group scoring. All tables should be numbered with Arabic numerals. Captions should be placed above tables, center justified. Tables must be embedded into the text and not supplied separately. Below is an example that authors may find useful.

There are many kinds of models of fuzzy numbers, which are mainly divided into triangular fuzzy numbers, trapezoidal fuzzy numbers, normal fuzzy numbers, parabolic fuzzy numbers, etc. In this paper, we mainly study the triangular fuzzy 
number, so we choose the triangular fuzzy number to describe the probability of occurrence of the bottom event. Triangular fuzzy number $A .=[\alpha, m, \beta]$, where $\mathrm{m}$ is the median and $\alpha$ and $\beta$ are the upper and lower of the triangular fuzzy number. In the absence of statistical data, $n$ experts estimate the probability of the occurrence of the basic events in the fault tree, and the results are expressed as $p_{1}, p_{2}, p_{3}, \cdots, p_{n}$. A triangular fuzzy number is constructed by the mean value and the variance of the estimated probability, shown as Equations (2) and (3) where $\mu$ represents the mean value and $\sigma$ represents the variance.

$$
\begin{gathered}
\mu=\frac{1}{n} \sum_{i=1}^{n} p_{i} \mu \\
\sigma=\sqrt{\frac{1}{n} \sum_{i=1}^{n}\left(p_{i}-\mu\right)^{2}}
\end{gathered}
$$

Suppose the expert estimates the probability value to follow the normal statistical rule. According to the $3 \sigma$ rule, the basic event whose probability exceeds $99.74 \%$ should fall in $[u-3 \sigma, u+3 \sigma]$. Therefore, we can set $m=u, \alpha=u-3 \sigma$, and $\beta=u+3 \sigma$. Obviously, the $m$ is determined, while the number between the lower $\alpha$ and the upper $\beta$ of the triangular fuzzy number is indefinite, and $\alpha$ and $\beta$ give the range of values. From this perspective, the triangular fuzzy number is the unity of certainty and uncertainty. It is confirmed that if the estimates are accurate, the upper and lower bounds of the fuzzy trigonometry can be determined using $u \pm \gamma \sigma$, which is determined by the investigators, $0<\gamma \leq 3$.

\subsection{The Basic Principle of Connection Number}

SPA (set pair analysis) was put forward by Zhao in 1989 [18]. SPA is a new theory of uncertainty. The core idea of SPA is to analyze the deterministic and uncertainty measures of objective things as a system, dealing with mixed uncertainty problems caused by fuzzy, random, unascertained, and mediating. The connection number is the main mathematical tool in the SPA. As a kind of connection number, the binary connection number is defined as follows:

Definition 1 Set $R$ as a real number set, then the binary connection number $0<a, b \in R, i \in[-1,1]$.

Definition 2 Addition operation, let two connection numbers $L_{1}=a_{1}+b_{1} i$ and $L_{2}=a_{2}+b_{2} i$ define the sum of two connection numbers $L=L_{1}+L_{2}$, which is denoted as Equation (4).

$$
L=L_{1}+L_{2}=\left(a_{1}+a_{2}\right)+\left(b_{1}+b_{2}\right) i=a+b i
$$

Definition 3 Multiplication operation, let two connection numbers $L_{1}=a_{1}+b_{1} i$ and $L_{2}=a_{2}+b_{2} i$ define two connection numbers $L=L_{1} \times L_{2}$, which is denoted as Equation (5).

$$
L=L_{1} \times L_{2}=a_{1} a_{2}+\left(a_{1} b_{2}+a_{2} b_{1}\right) i+b_{1} b_{2} i^{2}
$$

According to the theory of set pair analysis, without considering the level of uncertainty or the product of two connection numbers, the different powers of " $i$ " can be treated as a power, e.g.:

$$
L=L_{1} \times L_{2}=a_{1} a_{2}+\left(a_{1} b_{2}+a_{2} b_{1}+b_{1} b_{2}\right) i
$$

Let $a=a_{1} a_{2}, b=a_{1} b_{2}+a_{2} b_{1}+b_{1} b_{2}$, then $L=a+b i$. It can be proven that when there are three or more binary connection numbers multiplied, the product is still the binary number.

Definition 4 Division operation, it is known that $L=a+b i$ is the product of two binary connection numbers, one of which is $L_{1}=a_{1}+b_{1} i$, and the other is 


$$
L_{2}=\frac{L}{L_{1}}=\frac{a+b i}{a_{1}+b_{1} i}=\frac{a}{a_{1}}+\frac{b-a_{2} b_{1}}{a_{1}+b_{1}} i
$$

Let $a_{2}=a / a_{1}, b_{2}=\left(b-a_{2} b_{1}\right) /\left(a_{1}+b_{1}\right)$, then $L_{2}=a_{2}+b_{2} i$. It can be proven that when there are three or more binary connection numbers divided, the quotient is still the binary connection number.

\subsection{Transform Triangular Fuzzy Number into Binary Connection Number}

The triangular fuzzy number has the characteristics of certainty and uncertainty. The median of the triangular fuzzy number corresponds to the determined quantity of the connection number with $a=m$, the length of interval $(\beta-\alpha)$ in the triangular fuzzy number corresponds to the uncertainty of the connection number with $b=\beta-\alpha$, and then the triangular fuzzy number $L$ can be transformed into ( $a+b i)$ type connection number. The formula is shown as Equation (8).

$$
L=m+(\beta-\alpha) i=a+b i
$$

Equation (8) is called the conversion of transforming the triangular fuzzy number into the binary conversion number. $b=\beta-\alpha$ is called the difference degree of the connection number. The value range of $i$ is $[(\alpha-m) /(\beta-\alpha),(\beta-m) /(\beta-\alpha)]$. The connection number can be used to indicate the probability of occurrence of the bottom event.

$$
\tilde{F}_{k}=m_{k}+\left(\beta_{k}-\alpha_{k}\right) i=a_{k}+b_{k} i
$$

Once the probability of occurrence of the bottom event and the structure function of the top event are given, using the logic gate operator to calculate the probability of occurrence of the basic event, the failure probability of the top event is obtained. The calculation of the AND gate and OR gate represented by the connection number is shown in Equations (10) and (11), respectively.

$$
\begin{gathered}
\tilde{F}_{s}^{\text {and }}=\prod_{k=1}^{n} \tilde{F}_{k}=\tilde{F}_{1} \cdot \tilde{F}_{2} \cdots \cdots \tilde{F}_{n}=\prod_{k=1}^{n}\left[m_{k}+\left(\beta_{k}-\alpha_{k}\right) i\right]=\prod_{k=1}^{n}\left(a_{k}+b_{k} i\right) \\
\tilde{F}_{s}^{o r}=1-\prod_{k=1}^{n}\left(1-\tilde{F}_{k}\right)=1-\prod_{k=1}^{n}\left[1-m_{k}-\left(\beta_{k}-\alpha_{k}\right) i\right]=1-\prod_{k=1}^{n}\left(1-a_{k}-b_{k} i\right)
\end{gathered}
$$

The probability importance of the bottom event means that when only the $j$ event changes from the normal state to the failed state, the rate change of the probability of occurrence of the top event is:

$$
I_{h}(j)=\frac{\partial h(p)}{\partial p_{j}}, j=1,2, \cdots, n
$$

In Equation (12), $h(p)=h\left(p_{1}, p_{2}, \cdots, p_{n}\right)$ is the top event fuzzy fault function and $p_{j}$ is the fuzzy probability of the $X_{j}$ bottom event. This paper uses the contact number to describe the probability of occurrence of the bottom event to analyze the importance of the fuzzy probability of the basic event. The built fuzzy fault tree is composed of only the OR gate; therefore, the formula is shown as Equations (13) to (15).

$$
\begin{gathered}
\partial h(p)=1-\prod_{k=1}^{n}\left(1-\tilde{F}_{k}\right)=1-\prod_{k=1}^{n}\left[1-m_{k}-\left(\beta_{k}-\alpha_{k}\right) i\right]=1-\prod_{k=1}^{n}\left(1-a_{k}-b_{k} i\right) \\
\partial p_{j}=a_{k}+b_{k} i \\
I_{h}(j)=\prod_{k=1, k \neq j}^{n}\left(1-a_{k}-b_{k} i\right)
\end{gathered}
$$




\section{4. " $i$ "Value in the Connection Number}

In the comparison of the connection number, $i$ is based on the "principle of ratio of $i$ " [13]. The formula is shown as Equation (6).

$$
i=\frac{a_{k}}{a_{k}+b_{k}}
$$

Bringing the $i$ value into the top event with the connection number as the failure probability, we can calculate the top event failure probability value. Bringing the $i$ value into Equation (15), we can calculate the bottom event probability importance. Then, we can sort the risk of bottom event, find the key bottom event, and provide support for management decisions.

\section{Case Study}

\subsection{Build the Fault Tree}

This article takes a certain type of CNC machine tool spindle system as an example to verify the correctness of the proposed method. There is only one spindle in the headstock. Without the use of a transmission gear, only a set of triple V-belts is used to achieve step less speed change through an AC continuously variable motor. The spindle drives the spindle encoder synchronously through a pair of pulleys and toothed belts. The tightness of the toothed belt is regulated by the screw. A hydraulic cylinder is mounted on the rear end of the spindle to control the clamping and release of the hydraulic chuck on the spindle. The main shaft system consists of a main shaft, bearings, spindle box, chuck, belt, and proximity switches. Through the analysis of fault records, the main faults of the spindle system are shown in Table 1.

\begin{tabular}{|c|c||c|c|}
\hline Fault code & Fault name & Fault code & Fault name \\
\hline$X_{1}$ & Belt fault & $X_{9}$ & Bearing preload is too large \\
\hline$X_{2}$ & Spindle system fault & $X_{10}$ & Bearing grinding injury \\
\hline$X_{3}$ & Bearing galling & $X_{11}$ & Spindle does not drive \\
\hline$X_{4}$ & Large cutting vibration & $X_{12}$ & Bearing damage \\
\hline$X_{5}$ & Spindle overheating & $X_{13}$ & Bearing fault \\
\hline$X_{6}$ & Loose headstock connection screws & $X_{14}$ & Proximity switch fault \\
\hline$X_{7}$ & Bad bearing lubrication & $X_{15}$ & Bearing preload is not enough \\
\hline$X_{8}$ & Chuck is not tightened & & \\
\hline
\end{tabular}

The elements involved in the example system failure are $X_{1}, X_{2}, \cdots, X_{15}$, determined by designers, engineers, and maintenance staff to find the causality between the elements. The $(A+I)$ matrix is established as follows, and the reachable matrix $M$ is calculated by using Equation (1).

$$
A+I=\left[\begin{array}{lllllllllllllll}
1 & 1 & 0 & 0 & 0 & 0 & 0 & 0 & 0 & 0 & 0 & 0 & 0 & 0 & 0 \\
0 & 1 & 0 & 0 & 0 & 0 & 0 & 0 & 0 & 0 & 0 & 0 & 0 & 0 & 0 \\
0 & 0 & 1 & 0 & 0 & 0 & 0 & 0 & 0 & 0 & 0 & 0 & 1 & 0 & 0 \\
0 & 1 & 0 & 1 & 0 & 0 & 0 & 0 & 0 & 0 & 0 & 0 & 0 & 0 & 0 \\
0 & 1 & 0 & 0 & 1 & 0 & 0 & 0 & 0 & 0 & 0 & 0 & 0 & 0 & 0 \\
0 & 0 & 0 & 1 & 0 & 1 & 0 & 0 & 0 & 0 & 0 & 0 & 0 & 0 & 0 \\
0 & 0 & 0 & 1 & 0 & 0 & 1 & 0 & 0 & 0 & 0 & 0 & 0 & 0 & 0 \\
0 & 0 & 0 & 0 & 0 & 0 & 0 & 1 & 0 & 0 & 1 & 0 & 0 & 0 & 0 \\
0 & 0 & 0 & 0 & 1 & 0 & 0 & 0 & 1 & 0 & 0 & 0 & 0 & 0 & 0 \\
0 & 0 & 0 & 0 & 1 & 0 & 0 & 0 & 0 & 1 & 0 & 0 & 0 & 0 & 0 \\
0 & 1 & 0 & 0 & 0 & 0 & 0 & 0 & 0 & 0 & 1 & 0 & 0 & 0 & 0 \\
0 & 0 & 0 & 0 & 1 & 0 & 0 & 0 & 0 & 0 & 0 & 1 & 0 & 0 & 0 \\
0 & 0 & 0 & 1 & 0 & 0 & 0 & 0 & 0 & 0 & 0 & 0 & 1 & 0 & 0 \\
0 & 0 & 0 & 0 & 0 & 0 & 0 & 0 & 0 & 0 & 1 & 0 & 0 & 1 & 0 \\
0 & 0 & 0 & 0 & 0 & 0 & 0 & 0 & 0 & 0 & 0 & 0 & 1 & 0 & 1
\end{array}\right]
$$




$$
M=\left[\begin{array}{lllllllllllllll}
1 & 1 & 0 & 0 & 0 & 0 & 0 & 0 & 0 & 0 & 0 & 0 & 0 & 0 & 0 \\
0 & 1 & 0 & 0 & 0 & 0 & 0 & 0 & 0 & 0 & 0 & 0 & 0 & 0 & 0 \\
0 & 1 & 1 & 1 & 0 & 0 & 0 & 0 & 0 & 0 & 0 & 0 & 1 & 0 & 0 \\
0 & 1 & 0 & 1 & 0 & 0 & 0 & 0 & 0 & 0 & 0 & 0 & 0 & 0 & 0 \\
0 & 1 & 0 & 0 & 1 & 0 & 0 & 0 & 0 & 0 & 0 & 0 & 0 & 0 & 0 \\
0 & 1 & 0 & 1 & 0 & 1 & 0 & 0 & 0 & 0 & 0 & 0 & 0 & 0 & 0 \\
0 & 1 & 0 & 1 & 0 & 0 & 1 & 0 & 0 & 0 & 0 & 0 & 0 & 0 & 0 \\
0 & 1 & 0 & 0 & 0 & 0 & 0 & 1 & 0 & 0 & 1 & 0 & 0 & 0 & 0 \\
0 & 1 & 0 & 0 & 1 & 0 & 0 & 0 & 1 & 0 & 0 & 0 & 0 & 0 & 0 \\
0 & 1 & 0 & 0 & 1 & 0 & 0 & 0 & 0 & 1 & 0 & 0 & 0 & 0 & 0 \\
0 & 1 & 0 & 0 & 0 & 0 & 0 & 0 & 0 & 0 & 1 & 0 & 0 & 0 & 0 \\
0 & 1 & 0 & 0 & 1 & 0 & 0 & 0 & 0 & 0 & 0 & 1 & 0 & 0 & 0 \\
0 & 1 & 0 & 1 & 0 & 0 & 0 & 0 & 0 & 0 & 0 & 0 & 1 & 0 & 0 \\
0 & 1 & 0 & 0 & 0 & 0 & 0 & 0 & 0 & 0 & 1 & 0 & 0 & 1 & 0 \\
0 & 1 & 0 & 1 & 0 & 0 & 0 & 0 & 0 & 0 & 0 & 0 & 1 & 0 & 1
\end{array}\right]
$$

Table 2 represents the row sum of each element in the reachable matrix $M$, and Table 3 shows the sort by row sum in ascending order.

Table 2. Element row sum in the $M$ matrix

\begin{tabular}{|c|c|c|c|c|c|c|c|c|}
\hline element & $X_{1}$ & $X_{2}$ & $X_{3}$ & $X_{4}$ & $X_{5}$ & $X_{6}$ & $X_{7}$ & $X_{8}$ \\
\hline Row sum & 2 & 1 & 4 & 2 & 2 & 3 & 3 & 3 \\
\hline \hline element & $X_{9}$ & $X_{10}$ & $X_{11}$ & $X_{12}$ & $X_{13}$ & $X_{14}$ & $X_{15}$ & \\
\hline Row sum & 3 & 3 & 2 & 3 & 3 & 3 & 4 & \\
\hline
\end{tabular}

Table 3. The order of $M$ matrix element row sum
\begin{tabular}{|c|c|c|c|c|c|c|c|c|}
\hline element & $X_{2}$ & $X_{1}$ & $X_{4}$ & $X_{5}$ & $X_{11}$ & $X_{6}$ & $X_{7}$ & $X_{8}$ \\
\hline Row sum & 1 & 2 & 2 & 2 & 2 & 3 & 3 & 3 \\
\hline \hline element & $X_{9}$ & $X_{10}$ & $X_{12}$ & $X_{13}$ & $X_{14}$ & $X_{3}$ & $X_{15}$ & \\
\hline Row sum & 3 & 3 & 3 & 3 & 3 & 4 & 4 & \\
\hline
\end{tabular}

The $M^{\prime}$ matrix is obtained by adjusting the rows and columns of the $M$ matrix. The relationship between the two elements can be transferred by other elements to delete the leapfrog binary relationship in the $M^{\prime \prime}$, and then the skeleton matrix $M^{\prime \prime}$ can be obtained.

$$
M^{\prime}=\left[\begin{array}{lllllllllllllll}
1 & 0 & 0 & 0 & 0 & 0 & 0 & 0 & 0 & 0 & 0 & 0 & 0 & 0 & 0 \\
1 & 1 & 0 & 0 & 0 & 0 & 0 & 0 & 0 & 0 & 0 & 0 & 0 & 0 & 0 \\
1 & 0 & 1 & 0 & 0 & 0 & 0 & 0 & 0 & 0 & 0 & 0 & 0 & 0 & 0 \\
1 & 0 & 0 & 1 & 0 & 0 & 0 & 0 & 0 & 0 & 0 & 0 & 0 & 0 & 0 \\
1 & 0 & 0 & 0 & 1 & 0 & 0 & 0 & 0 & 0 & 0 & 0 & 0 & 0 & 0 \\
1 & 0 & 1 & 0 & 0 & 1 & 0 & 0 & 0 & 0 & 0 & 0 & 0 & 0 & 0 \\
1 & 0 & 1 & 0 & 0 & 0 & 1 & 0 & 0 & 0 & 0 & 0 & 0 & 0 & 0 \\
1 & 0 & 0 & 0 & 1 & 0 & 0 & 1 & 0 & 0 & 0 & 0 & 0 & 0 & 0 \\
1 & 0 & 0 & 1 & 0 & 0 & 0 & 0 & 1 & 0 & 0 & 0 & 0 & 0 & 0 \\
1 & 0 & 0 & 1 & 0 & 0 & 0 & 0 & 0 & 1 & 0 & 0 & 0 & 0 & 0 \\
1 & 0 & 0 & 1 & 0 & 0 & 0 & 0 & 0 & 0 & 1 & 0 & 0 & 0 & 0 \\
1 & 0 & 1 & 0 & 0 & 0 & 0 & 0 & 0 & 0 & 0 & 1 & 0 & 0 & 0 \\
1 & 0 & 0 & 0 & 1 & 0 & 0 & 0 & 0 & 0 & 0 & 0 & 1 & 0 & 0 \\
1 & 0 & 1 & 0 & 0 & 0 & 0 & 0 & 0 & 0 & 0 & 1 & 0 & 1 & 0 \\
1 & 0 & 1 & 0 & 0 & 0 & 0 & 0 & 0 & 0 & 0 & 1 & 0 & 0 & 1
\end{array}\right]
$$




$$
M^{\prime \prime}=\left[\begin{array}{llllllllllllllll}
1 & 0 & 0 & 0 & 0 & 0 & 0 & 0 & 0 & 0 & 0 & 0 & 0 & 0 & 0 \\
1 & 1 & 0 & 0 & 0 & 0 & 0 & 0 & 0 & 0 & 0 & 0 & 0 & 0 & 0 \\
1 & 0 & 1 & 0 & 0 & 0 & 0 & 0 & 0 & 0 & 0 & 0 & 0 & 0 & 0 \\
1 & 0 & 0 & 1 & 0 & 0 & 0 & 0 & 0 & 0 & 0 & 0 & 0 & 0 & 0 \\
1 & 0 & 0 & 0 & 1 & 0 & 0 & 0 & 0 & 0 & 0 & 0 & 0 & 0 & 0 \\
0 & 0 & 1 & 0 & 0 & 1 & 0 & 0 & 0 & 0 & 0 & 0 & 0 & \oint & 0 \\
0 & 0 & 1 & 0 & 0 & 0 & 1 & 0 & 0 & 0 & 0 & 0 & 0 & \oint & 0 \\
0 & 0 & 0 & 0 & 1 & 0 & 0 & 1 & 0 & 0 & 0 & 0 & 0 & \oint & 0 \\
0 & 0 & 0 & 1 & 0 & 0 & 0 & 0 & 1 & 0 & 0 & 0 & 0 & \oint & 0 \\
0 & 0 & 0 & 1 & 0 & 0 & 0 & 0 & 0 & 1 & 0 & 0 & 0 & \oint & 0 \\
0 & 0 & 0 & 1 & 0 & 0 & 0 & 0 & 0 & 0 & 1 & 0 & 0 & \oint & 0 \\
0 & 0 & 1 & 0 & 0 & 0 & 0 & 0 & 0 & 0 & 0 & 1 & 0 & \oint & 0 \\
0 & 0 & 0 & 0 & 1 & 0 & 0 & 0 & 0 & 0 & 0 & 0 & 1 & \oint & 0 \\
0 & 0 & 0 & 0 & 0 & 0 & 0 & 0 & 0 & 0 & 0 & 1 & 0 & 1 & 0 \\
0 & 0 & 0 & 0 & 0 & 0 & 0 & 0 & 0 & 0 & 0 & 1 & 0 & 0 & 1
\end{array}\right]
$$

In the skeleton matrix $M^{\prime \prime}$, from the upper left corner to the lower right corner, a unit matrix decomposes the maximum order in turn. Elements in the same unit matrix belong to the same level. The remaining "1" indicates the causal relationship between the elements. According to the " 1 " element in the intersection of the two square boxes in $M$ ", draw an interpretative structural model that represents the causal relationship between elements at different levels in Figure 2.

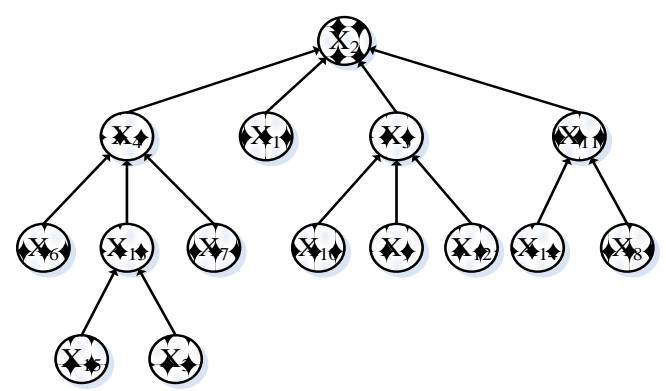

Figure 2. Interpretative structure model

The top event, the middle event, and the bottom event are all expressed in terms of elements when establishing the interpretive structure modeling. When the interpretative structure modeling is transformed into a fault tree, it transforms the elements into corresponding events according to the relationship between the levels, positions, and elements of the elements. The conversion rule states that only the elements pointed by the arrow are transformed into the top event, the elements not pointed by the arrow are transformed into bottom events, and the remaining elements are transformed into middle events. Translate the interpretive structure modeling into a fault tree as explained by Figure 2. The element $X_{2}$ is the top event denoted by $\mathrm{T}$, the elements $X_{1}, X_{6}, X_{7}, X_{10}, X_{9}, X_{12}, X_{14}, X_{8}, X_{15}$, and $X_{3}$ are the bottom events, and $X_{4}, X_{5}, X_{11}, X_{13}$ are the middle events that are represented by $M_{1}, M_{2}, M_{3}$, and $M_{4}$. Logic gates between events are determined by designers, engineers, and maintenance personnel based on the actual situation. The fault tree is shown in Figure 3.

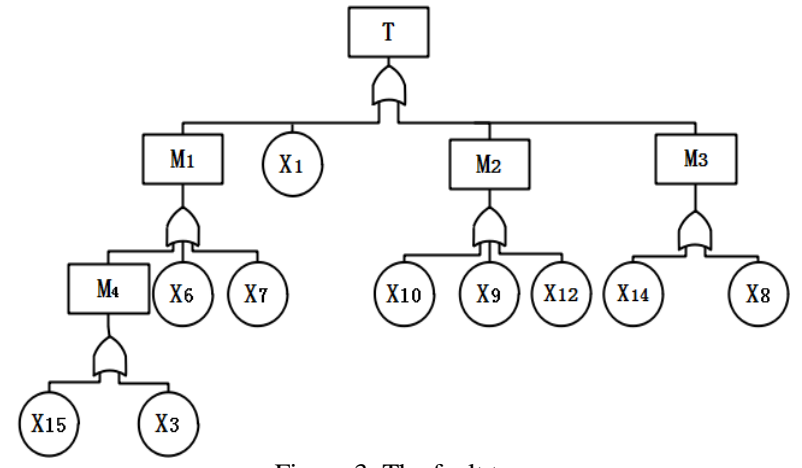

Figure 3. The fault tree

Once the fault tree is established, according to the logic gate operation rules, the minimum cut set can be found as: 
$\left\{X_{1}\right\},\left\{X_{6}\right\},\left\{X_{7}\right\},\left\{X_{9}\right\},\left\{X_{10}\right\},\left\{X_{12}\right\},\left\{X_{15}\right\},\left\{X_{3}\right\},\left\{X_{14}\right\},\left\{X_{8}\right\}$

\subsection{Quantitative Calculation}

The probabilities of each bottom event are estimated by the expert scoring method. The mean and variance of probability are calculated by Equations (2) and (3). The triangular fuzzy number of the bottom event probability is shown in Table 4 .

Table 4. Bottom event triangle fuzzy probability

\begin{tabular}{|c|c||c|c|}
\hline $\begin{array}{c}\text { Bottom } \\
\text { event }\end{array}$ & TFM & $\begin{array}{c}\text { Bottom } \\
\text { event }\end{array}$ & TFM \\
\hline$X_{1}$ & $(0.014,0.016,0.018)$ & $X_{9}$ & $(0.022,0.034,0.046)$ \\
\hline$X_{3}$ & $(0.014,0.017,0.020)$ & $X_{10}$ & $(0.050,0.060,0.070)$ \\
\hline$X_{6}$ & $(0.018,0.022,0.026)$ & $X_{12}$ & $(0.038,0.040,0.042)$ \\
\hline$X_{7}$ & $(0.008,0.011,0.014)$ & $X_{14}$ & $(0.005,0.007,0.009)$ \\
\hline$X_{8}$ & $(0.018,0.03,0.042)$ & $X_{15}$ & $(0.040,0.050,0.060)$ \\
\hline
\end{tabular}

Transform triangular fuzzy numbers into binary relation numbers using Equation (7). The conversion results are shown in Table 5.

Table 5. Binary connection number of bottom event

\begin{tabular}{|c|c||c|c|}
\hline $\begin{array}{c}\text { Bottom } \\
\text { event }\end{array}$ & BCN & $\begin{array}{c}\text { Bottom } \\
\text { event }\end{array}$ & BCN \\
\hline$X_{1}$ & $0.016+0.004 i$ & $X_{9}$ & $0.034+0.024 i$ \\
\hline$X_{3}$ & $0.017+0.006 i$ & $X_{10}$ & $0.060+0.020 i$ \\
\hline$X_{6}$ & $0.022+0.008 i$ & $X_{12}$ & $0.040+0.004 i$ \\
\hline$X_{7}$ & $0.011+0.006 i$ & $X_{14}$ & $0.007+0.004 i$ \\
\hline$X_{8}$ & $0.030+0.024 i$ & $X_{15}$ & $0.050+0.020 i$ \\
\hline
\end{tabular}

\subsection{Calculation of Top and Bottom Event Failure Probability}

The fuzzy failure probability of the top event is calculated by Equation (9), $L T=0.2537+0.0882 i$, the exact failure probability of the top event is calculated by Equation (14), and the result is 0.3194 . The probability importance of the bottom event is calculated by Equation (13) and shown in Table 6. The exact probability value of the base event is calculated by Equation (14) and shown in Table 7. Bottom event exact values are sorted, and the sort result is $X_{10}, X_{15}, X_{9}, X_{8}, X_{12}, X_{6}, X_{3}, X_{1}, X_{7}, X_{14}$. Find and control the major events of system failure.

Table 6. Probability importance of bottom event

\begin{tabular}{|c|c||c|c|}
\hline $\begin{array}{c}\text { Bottom } \\
\text { event }\end{array}$ & $I_{(h)}(j)$ & $\begin{array}{c}\text { Bottom } \\
\text { event }\end{array}$ & $I_{(h)}(j)$ \\
\hline$X_{1}$ & $0.7584-0.0869 i$ & $X_{9}$ & $0.7726-0.0739 i$ \\
\hline$X_{3}$ & $0.7592-0.0856 i$ & $X_{10}$ & $0.7939-0.0786 i$ \\
\hline$X_{6}$ & $0.7631-0.0846 i$ & $X_{12}$ & $0.7774-0.0890 i$ \\
\hline$X_{7}$ & $0.7546-0.0851 i$ & $X_{14}$ & $0.7515-0.0861 i$ \\
\hline$X_{8}$ & $0.7694-0.0737 i$ & $X_{15}$ & $0.7856-0.0779 i$ \\
\hline
\end{tabular}

Table 7. Exact probability value of bottom event

\begin{tabular}{|c|c||c|c|}
\hline $\begin{array}{c}\text { Bottom } \\
\text { event }\end{array}$ & $I_{(h)}(j)$ & $\begin{array}{c}\text { Bottom } \\
\text { event }\end{array}$ & $I_{(h)}(j)$ \\
\hline$X_{1}$ & 0.6804 & $X_{9}$ & 0.7052 \\
\hline$X_{3}$ & 0.6823 & $X_{10}$ & 0.7224 \\
\hline$X_{6}$ & 0.6869 & $X_{12}$ & 0.6975 \\
\hline$X_{7}$ & 0.6781 & $X_{14}$ & 0.6743 \\
\hline$X_{8}$ & 0.7021 & $X_{15}$ & 0.7147 \\
\hline
\end{tabular}




\section{Conclusions}

In this paper, interpretive structure modeling combined with a hierarchical structure digraph method was investigated to build a fault tree. This paper proved that triangular fuzzy numbers can also be converted into binary connection numbers. This not only simplifies the calculation process, but also explore a new way of triangular fuzzy numbers usage. In future works, the reliability based topology for the motor will be investigated and applied when the constant force is observed. The determination of the " $i$ " value in the binary connection number will also be a focus of future research.

\section{Acknowledgements}

This work was partially supported by the National Natural Science Foundation of China (No. 71761030).

\section{References}

1. J. X. Hu, L. Y. Xie, Y. Xin, X. Bai, and E. J. Bai, "FMECA-based FTA Automatic Fault Tree Drafting," Journal of Harbin Engineering University, Vol. 38, No. 7, pp. 1162-1166, July 2017

2. Z. H. Du and C. C. Di, "Mechanism Product Integrated Study of FTA and FMECA based on VB and ACCESS," Computer Applications Technology, Vol. 36, No. 10, pp. 34-38, October 2009

3. J. F. Tao, S. P. Wang, and S. P. Du, "Fault Diagnosis for Clutch and Brake based on Expert System," Journal of Beijing University of Aeronautics and Astronautics, Vol. 26, No. 6, pp. 663-665, December 2000

4. W. H. Xu and Y. P. Zhang, "A Fault Tree Auto-Modeling Method based on Avionics System Architecture Model," Computer Engineering \& Science, Vol. 39, No. 12, pp. 2269-2277, December 2017

5. P. Yang, "Research on Method of Fault Tree Created Automatically based on FMECA," Computer and Modernization, No. 12, pp. 193-196, December 2009

6. M. Y. Huang, O. Wei, and J. Hu, "Fault Tree Generation based on Fault Configuration," Computer Science, Vol. 44, No. 2, pp. 182-191, February 2017

7. M. Li, C. P. Cao, and Y. Sun, "Fault Diagnosis for Clutch and Brake based on Expert System," Forging \& Stamping Technology, Vol. 42, No. 12, pp. 163-169, December 2017

8. W. H. Gu and S. G. Wang, "Collapse Risk Analysis for the Loess-featured Tunnels in Railway Construction based on ISM and Fuzzy Fault Tree Method," Computer Science, Vol. 44, No. 2, pp. 182-191, October 2017

9. R. S. Sun and X. Zhan, "Analysis on Influence Factors of Controlled Flight into Terrain of General Aviation based on ISMCRITIC Method," Journal of Safety Science and Technology, Vol. 14, No. 1, pp. 129-135, January 2018

10. Y. M. Wei, X. S. Gan, W. W. You, and M. L. Jiang, "Inducement Relationship Analysis of Controlled Flight into Terrain based on ISM Model," Fire Control \& Command Control, Vol. 43, No. 2, pp. 172-176, February 2018

11. J. H. Xia and H. J. Wang, "Fault Cause Analysis of Complex Manufacturing System based on DEMATEL-ISM," Journal of Beijing Information Science \& Technology University, Vol. 33, No. 1, pp. 31-47, February 2018

12. H. Z. Huang and Y. F. Li, "A New Ordering Method of based Events in Fault Tree Analysis," Quality and Reliability Engineering International, Vol. 28, No. 3, pp. 297-305, March 2012

13. Z. S. Xu, "Study on Method for Triangular Fuzzy Number based Multi-attribute Decision Making with Preference Information on Alternatives," Systems Engineering and Electronics, Vol. 124, No. 18, pp. 9-12, August 2002

14. C. B. Li and Z. Y. Pan, "Fuzzy Comprehensive Assessment of Power Enterprise R\& D Risk based on Triangular Fuzzy Number," Journal of Chongqing University of Technology (Natural Science), Vol. 31, No. 1, pp. 143-151, January 2017

15. C. Q. Zhao, J. W. Zhang, Y. Sun, and Y. Y. Zhang, "Analysis and Application of Fire and Explosion Fault Tree in Hydrocracking Unit based on Triangular Fuzzy Number," Safety and Environmental Engineering, Vol. 24, No. 6, pp. 119-122, November 2017

16. X. C. Li, Q. L. Liu, and L. S. Pei, “Analysis on Danger Sources of Mine Gas Explosion based on Fuzzy Fault Tree,” Coal Engineering, Vol. 46, No. 5, pp. 93-96, May 2014

17. L. Yang and G. H. Qi, "A Group Decision Making Method with Interval-Valued Triangular Fuzzy Attribute and Weight," Fuzzy Systems and Mathematics, Vol. 31, No. 1, pp. 179-186, February 2017

18. X. M. Liu, K. Q. Zhao, and C. B. Wang, "New Multiple Attribute Decision-Making Model with Triangular Fuzzy Numbers based on Connection Numbers," Systems Engineering and Electronics, Vol. 31, No. 10, pp. 2399-2403, October 2009

19. X. M. Liu and K. Q. Zhao, "Triangular Fuzzy Number Multi-Attribute Decision-Making with the Attribute Weight Unknown based on Connection Number," Fuzzy Systems and Mathematics, Vol. 31, No. 2, pp. 95-106, April 2017

20. G. D. Lu and C. Wu, "Interval Number Multiple-Attribute Decision-Making based on Adjoint Functions of Connection Number," Fuzzy Systems and Mathematics, Vol. 32, No. 2, pp. 182-190, February 2018

21. Y. L. Zhao and K. Q. Zhao, "Fault Diagnosis of Turbine based on Multiple Dual Connection Number," Modern Manufacturing Engineering, No. 2, pp. 155-160, February 2016

22. Y. L. Wang, "Systems Engineering," China Machine Press, Beijing, 2008

Honghua Sun is an associate professor in the School of Mechanical Engineering at Inner Mongolia University of Technology. She received her M.S. degree from Harbin Institute of Technology in 2008. Her current research interests 
include reliability data analysis and reliability evaluation.

Hongxia Chen is an associate professor in the School of Mechanical Engineering at Inner Mongolia University of Technology. She received her M.S. degree in 2008. Her current research interests include reliability data analysis and reliability evaluation.

Qingyang Li is a postgraduate student in the School of Mechanical Engineering at Inner Mongolia University of Technology. He received his bachelor's degree from Inner Mongolia University of Technology in 2016. His current research interests include reliability data analysis and reliability evaluation.

Xudong Chen is a postgraduate student in the School of Mechanical Engineering at Inner Mongolia University of Technology. He received his bachelor's degree from Inner Mongolia University of Technology in 2016. His current research interests include reliability data analysis and reliability evaluation. 